\title{
BIG DATA ANALYTICS WITH IoT: LARGE GEO GRAPHICAL INFRASTRUCTURE DEVELOPEMENT USING DIGITAL COMMUNICATION TECHNOLOGY
}

\author{
Boggadi Nagarjuna Reddy \\ Department of ECE \\ BIHER, Chennai, Tamil Nadu, India
}

\begin{abstract}
The web is without interruption developed gradually and become different. The IoT could be opinion is more about the web implementations are included with direct communication between devices are using either wired or wireless communication. The most of developers are worked and interested with infrastructure communication network and sensor technology because this technology is reached up to world secure technology. And this technology is seems like a human two eyes. The data implemented and linked with the devices are well being of web Internet of Things could be comfortably received. The usage of Internet of Things technology is more in different kind of areas like industrial automation, medical equipments, smart homes and smart grids. Most of well-being response is occurred because of the combination of Big Data analytics with internet of things but in here small intrinsic issues and errors are involved. This paper is mainly concentrate on the Big Data analytics and Internet of Things.
\end{abstract}

Keywords; IoT, Big Data Analytics, fog or edge computing, sensor technology etc.

\section{INTRODUCTION}

Now day's web usages are very closely related with human's life. The present trend is follows social media, webmail's and online shopping's on web, it's very simple process in web. The web technology is smoothly and slowly combines with purely human's regular life. The digital world is giving to the opportunity to improve the different kind of complex issues in different platforms. According to the result the number of different categories of sensors and electronic devices are connected with different parts but the information is transferring and sending because of "Internet of Things" [2]. The web generating almost all information to humans and saved the lot of information in storage devices, and interact with linked people to develops the quality of information. For that of accuracy most of viewed Internet of Things at different categories likes medical equipments and industrial automation
[5]. The Internet of Things is included with medical cure instrumentation the combination of success is proved results in human's world and health made organizations [7]. For accurate results tracking chip was executed in medical systems to track essential quality of the result for patient. Because of the quality of the essential results assist to designate whether its condition is good or bad.

Internet of Things is operated and controlled device and also protects the household devices and interact and operated with systematic life utilization. This is qualified to interacting at different energy components are frequently mentioned to direct communication between the devices using either wired or wireless channel. The IoT solid state components are easy to handle and controlled, humans are easily interact and knows very well where it's placed position. Components for example, containers or crafts taken in to consideration the most extreme ability will be loaded with correspondence among components and afterward handing-off that data to an individual to benefit from the information provided. One more favourable position is available in Internet of Things are monitor the customers information independently and also focusing the more number of customers because of these data is provided to the components. In that manner, using that designed methodology to improve the certain marketing area to improve the purchases and also improved the particular group of business. The different usages with Internet of Things are viewed in figure.1. Internet of Things is not only assigned with electronic components and also seeking peoples mindset how it will interact, evaluate and feelings. And this is also advantage of the Internet of Things but $\mathrm{n}$ here to generate primary issues and provocations. The significant deal is connected with Internet of Things are intricacy of the framework, area, capacity, safety and protection. Because of a mutual connection between two or more connections that is a unordinary chance to improve the complication of the structure. The web of the devices are converted in to coded form of 60 to 100 a million of devices should be means travel for the variation of that devices [9]. The span of Internet of Things should be a noteworthy concern. The information 
escalated creation of Internet of Things could be diverted with Big Data as a piece of the answer for the difficulties looked by Internet of Things.

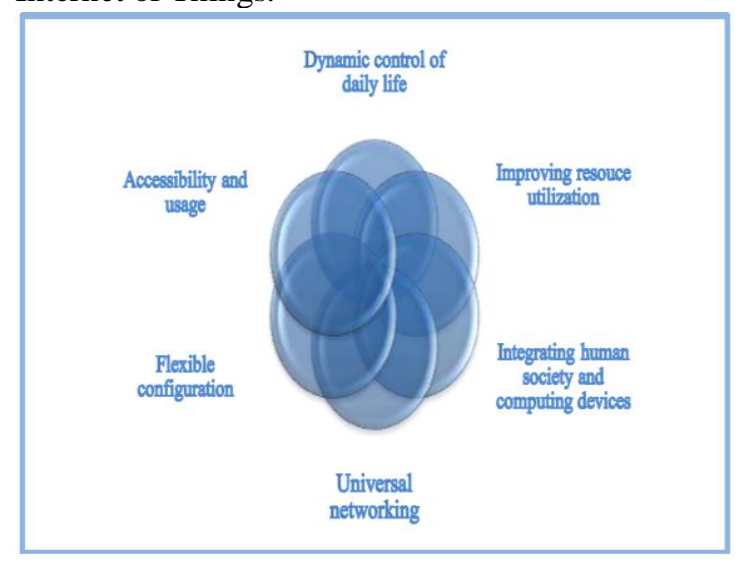

Figure.1. The organization of IoT elements.

The sending of different instruments is likewise adding with that storm of information or present additional transcendently called as large data. The Internet of Things is best significant hotspot for Big Data. When tally of different parts are connected to the instruments and supporting to expanding the information and it's related to the setting of the enormous identical. The Internet of Things are divide by passing through the Big Data and that information is too clear to developing that two technologies. The equality meaning of these big Data with internet of Things is billion of electronic devices are connected with internet. The area of the digital electronics world of more devices are inter linked with Internet of Things to be intensified. And this significant information isn't existence tribute [9].the Big Data Analysis is create the new methodology with good operating system further improve quality and secure and having practical information to the enormous information actuality amplified. These technology and paper purely maintain the concentration on Big Data with Internet of Things.

\section{DATA INTENSIVE IOT}

The big data analysis is methodology is created in particular manner or agreement between a buyer and a seller to exchange goods structure. The Internet of Things is providing a sequence of data elements made available over a time of information is same as to online platform. The technique of Internet of Things are imagined with brilliant distribution centre of the information is actuality put away with respect to the starting of the entryway of stockroom as term, heat, hour and 0 'clock, recurrence every period of $60 \mathrm{~min}$, out of each day, of the week. These IoT devices are unstoppable receiving of information and that reality is monitored with located sensors. In this same manner the utilization of brilliant houses matters as a rooftop harm, rain and liquid spillage, energy utilization is controlled with sensors and electronic instruments, the information is continuously monitoring the exact values to minute to minute. Because of this structure of execution is represents large response is occurred when the Big Data is combines with Internet of Things. In here this technology is reading the analytical information and generates practically monitored information.

\section{IOT WITH BIGDATA ANALYSIT}

The information is received from the electronic computing devices in that information removes the unwanted information and taken the helpful information. The reality of the excess information is gathered is overwhelming because of the pure idea to the structure of Internet of Things. The information is opposing the un interrupted information of significant data isn't basic. It needs a perfect instruments of systematic rules and operating system is protect that information is safely and consequential [17]. And that information is received from the electronic computing components and these components are receiving and sending the information to controllable service in a network. In this same manner the information is also transmitted return to the components. The system is to be ideal because of this execution proficiency. Internet of Things are dealing with the multiple technologies that are Embedded Systems, Machine learning, Commodity sensors, Control systems and Automation. All these technologies are monitoring the information, controls the devices operation and sending and receiving the information and also improve the system problems and also maintain the pure information.

In people's minds are having the both Big Data and Internet of Things are different and that are the both aspect of a result of perceiving. Dealing with the information and extricating data and its an extremely indispensable errand related with Internet of Things. The suitable systematic methodology is needed to authorize to gain the practical understanding of the Internet of Things information. The IoT components are to produces un interrupted continuous flow of information in a climbed manner. And the necessary to maintained a large capacity of continuous flow of information otherwise hackers are attack on the information. The continuous flow of information is available in Internet of Things and not in Big Data. But using Big Data with IoT it's not easy to hack that information some actions are available in this technology. For information monitoring and storage purpose Internet of Things are best option for real time systems. Continuous Big Data investigation and IoT compares to esteem creation as shown in fig. 2 .

In here to execute the continuous statistics with an Internet of Things atmosphere is demanding because of these issues. The massive count of electronic computing devices is utilized and also produces bulk of information. The information monitoring is very essential at statistics of the information and 
also the delay of the transfer information begins to allowing an instruction for transfer. And also expecting importance of quality monitoring and value of the purity data. Specially needed software subsystems or internet of things to design a perfect methodology and application satisfied strategy. The systematic computational advanced analysis on internet of things storage information is very useful to scan the information up to certain long period and its to gain an accurate and deep understanding in monitoring devices and their information properties.

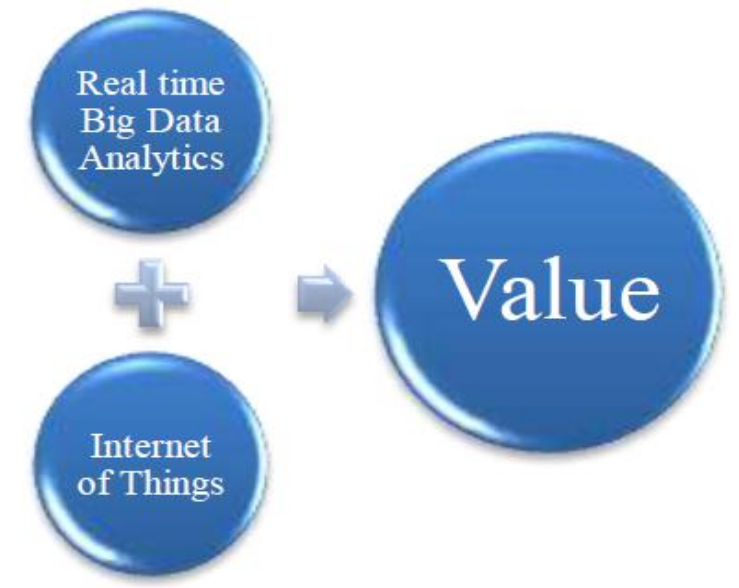

Figure.2.Value Creation.

To design an advanced methodology results and to reduce the equivalent internet of things [18]. The gathering data and units of information is not calculated straight through sensors, in this way of manner the different kind of internet of things parameters are influenced on other parameters.

\section{THEMATIC ANALYSIS}

The thematic analysis is improved based on two ways of implementation. The first way is to develop the technology and methodologies for advanced development applications using internet of things are applicable on environmental depletion or degradation of natural resources and other cases. The second way is based on to design and improvement of theoretical analysis on smart cities and in here using advanced technological devices and components, these are associated with environment ability to maintain certain level efficiency of system devices and its reduce the environmental issues based on this information. in this review mainly concentrate on the smart cities, information gathered device, information calculated or secured methods and different kind of actionable policies based on environmental situations. This technology most commonly used for weather checking, pollution control purpose, and particular city security purposes. In this application process is interlinked with so many technologies are sensors advanced technology, data analytics and different computing methods. The automated recognition of patterns and regularities in information and evidently to see the repeated information. And once again check in similarities of the information in patterns based on codes concepts. The thematic analysis Practically connected to the all the hospitals organisations like health care experts, medical researchers, governments and specialized companies, in health care organisations are improved based on computational structures and systems, these are maintained a purely successes plans and implemented with practical proof. In a joint effort continue to our insight computational users, targeting users are planned to comprehend made in a particular way of these systems compelling, and find out systems drew in their partners and what information interpretation and commitment resembled over their drives

The phase 1 represents the qualitative information collecting from different ways like live recording information, stored information, messaging, voice calls, documents, and images. The phase 2 mainly focused on the generating of coding using pahse1 stored information, based on this coding information easy to find out the repeated information and remove the reflected information for coding accuracy. This coding is very easy to concentrate on particular specialized of the information. the phase 3 is at starting different kind of information's are coded and collect and combined with text, information or data, and in here different set of information codes are identified using all data set and improve the coding response. This phase mainly coding is arranged systematically in different groups and collect and combine all the quality coded data is extracts in to themes. The phase 4 represents at a time number of themes are very complex procedure, and in this process need removing of unnecessary elements. In this phase 5 the researchers are theme captured on the particular data, indentified and why concentrating on particular data why. in this process researchers are need to write a complete analysis on each and particular theme, these analysis gives clarity about theme story. The last phase researchers are implemented total themes and The detailed information of thematic analysis mostly provide a logical, coherent, concise, no repetitive, using these all create a data account and across themes. This process of methods and analysis is find outing the theoretical relationships for future development of smart cities. 
Table I. Establishing Trustworthiness During Each Phase of Thematic Analysis.

\begin{tabular}{|c|c|}
\hline $\begin{array}{l}\text { Phases of Thematic } \\
\text { Analysis }\end{array}$ & Means of Establishing Trustworthiness \\
\hline $\begin{array}{l}\text { Phase I: Familiarizing } \\
\text { yourself with your data }\end{array}$ & $\begin{array}{l}\text { Prolong engagement with data } \\
\text { Triangulate different data collection } \\
\text { modes } \\
\text { Document theoretical and reflective } \\
\text { thoughts } \\
\text { Document thoughts about potential } \\
\text { codes/themes } \\
\text { Store raw data in well-organized archives } \\
\text { Keep records of all data field notes, } \\
\text { transcripts, and reflexive journals }\end{array}$ \\
\hline $\begin{array}{l}\text { Phase 2: Generating initial } \\
\text { codes }\end{array}$ & $\begin{array}{l}\text { Peer debriefing } \\
\text { Researcher triangulation } \\
\text { Reflexive journaling } \\
\text { Use of a coding framework } \\
\text { Audit trail of code generation } \\
\text { Documentation of all team meeting and } \\
\text { peer debriefings }\end{array}$ \\
\hline $\begin{array}{l}\text { Phase 3: Searching for } \\
\text { themes }\end{array}$ & $\begin{array}{l}\text { Researcher triangulation } \\
\text { Diagramming to make sense of theme } \\
\text { connections } \\
\text { Keep detailed notes about development } \\
\text { and hierarchies of concepts and } \\
\text { themes }\end{array}$ \\
\hline Phase 4: Reviewing themes & $\begin{array}{l}\text { Researcher triangulation } \\
\text { Themes and subthemes vetted by team } \\
\text { members } \\
\text { Test for referential adequacy by } \\
\text { returning to raw data }\end{array}$ \\
\hline $\begin{array}{l}\text { Phase 5: Defining and } \\
\text { naming themes }\end{array}$ & $\begin{array}{l}\text { Researcher triangulation } \\
\text { Peer debriefing } \\
\text { Team consensus on themes } \\
\text { Documentation of team meetings } \\
\quad \text { regarding themes } \\
\text { Documentation of theme naming }\end{array}$ \\
\hline $\begin{array}{l}\text { Phase 6: Producing the } \\
\text { report }\end{array}$ & $\begin{array}{l}\text { Member checking } \\
\text { Peer debriefing } \\
\text { Describing process of coding and analysis } \\
\text { in sufficient details } \\
\text { Thick descriptions of context } \\
\text { Description of the audit trail } \\
\text { Report on reasons for theoretical, } \\
\text { methodological, and analytical choices } \\
\text { throughout the entire study }\end{array}$ \\
\hline
\end{tabular}

\section{RELATED WORK}

In here to expand the theoretical and analytical literature is provided in section wise explained detailed review and technical analysis on big data analytics associated with IoT and sensor technology, this technology using different strategies on infrastructure city development.

\section{DATA ANALYTICS WITH HADOOP PLATFORM}

In here to advanced technology to developed based on realizing computing with smart devices of internet things and also to improved the techniques in big data analysis for collection of more and advanced information from smart cities [30,35]. The big data analytics is most commonly is used for different kind of applications like urban area developments, transport, traffic and power grids, energy and accessibility, these all applications are interlinked with environmental control pattern[15,16]. The information and communication technology infrastructure and computing systems are used in smart cities particularly with intent of things ecosystems. Its mainly focusing on optimization and control of automation management and urban computing systems organisation process and improvement of efficiency. And exclusively process of development of environmental methods and planning's are modified based on new technological trends, these are gradually increase the working functions, reduce the data utilization and decrease the environmental issues. The big data analytics application oriented base is taken different kind of components, algorithms, software tools and platforms are given proper response of data mining in this process have large data sets and methods like technique, data, knowledge view and application view, these all are related with the business intelligence. This research process design application oriented advanced computing systems, data analysing systems information is serial processing from input, the machine learning is automatically accesses the information and increase the quality of being efficient, graph processing and batch processing. The advanced software's and technology used to design large storage data devices.

The big data analysis main focused research on exact and good accurate on data operations are focused on different directions and also improve mining algorithm is very useful in big data analytics, its gives solutions for different issues in research point of view [30]. In previous researchers discussed and solved different issues on big data concentrated issues, software's, properties and inputs are implemented for different kind of applications, such as sensor information storage and social media improving.

\begin{tabular}{||l||l||}
\hline \hline HDFS & $\begin{array}{l}\text { Distributed file system } \\
\text { Subject of this paper! }\end{array}$ \\
\hline \hline MapReduce & Distributed computation framework \\
\hline \hline HBase & Column-oriented table service \\
\hline \hline Pig & $\begin{array}{l}\text { Dataflow language and parallel execution } \\
\text { framework }\end{array}$ \\
\hline \hline Hive & Data warehouse infrastructure \\
\hline \hline ZooKeeper & Distributed coordination service \\
\hline \hline Chukwa & System for collecting management data \\
\hline \hline Avro & Data serialization system \\
\hline
\end{tabular}

Table.3. hadoop components.

The hadoop components are very useful to develop big data analytics and algorithmic and programming techniques easy to implement and get in to the particular results already proved in big data analytics using hadoop infrastructure is easy to find out the locality of data and information is compressed within short of time. Because of in this review has so many advanced 
proposed technologies are linked with big data analytics. The computational and analytical technologies mainly concentrating on the particular set of procedure, main theme is information sensing and distribution is using different methodologies and techniques are easy to store the information and easy to maintain servers. Information distribution is only on data analytics computer systems using advanced software tools and database. The algorithms and analysis methods are in machine learning, statistics, and database systems operations. The wireless technology is one of the most important element to design to this work, GPS information networks, cell phones and WAN networks are very need to share information and collection of information. The information display process is taken smart electronic and intent of things, for displaying of valuable information.

\section{ANALYSIS OF SMART CITY ORGANAISATION.}

The structural representation of smart city framework as shown in below fig.4. in here to improve the integrative framework, this frame work methods and analysis is influenced on smart city initiatives, because of this effective framework mentioned. The framework elements all are very important for researchers and its helpful to improve city analysis one by one process. These elements are used to compare previous city developments, methods, implemented services and related challenges. The elements all are implemented using different software tools and computational systems, different way of processing's and different purposes. And the same manner this framework is very useful to developed smart cities in advance, this is mostly effect on different variables like organizational, technical and contextual, based on this easy to develop initiatives in smart city. In here most commonly expected thing is all the elements influenced with one element to other elements factors at the initial stage of researchers, and effected many ways with unnecessary contexts, some more having great influence on someone than others. The proposed framework reflects sometimes with elements with many ways of influence. The mentioned outer elements are city infrastructure, natural environment, peoples and economy, these all are influenced more than inner factors like technology, management and policy, and these are most affected elements to smart city paln implement. The collection of elements are affected with other situations either direct or indirect way. This technology most of times accept as network to implement in smart city ability to assess and initiate things independently, past developed analysis and methods most commonly influence with seven elements. For smart city development purpose most commonly prefer different kind of technologies, because of this way of implementation technology techniques leads on success based on these elements framework.

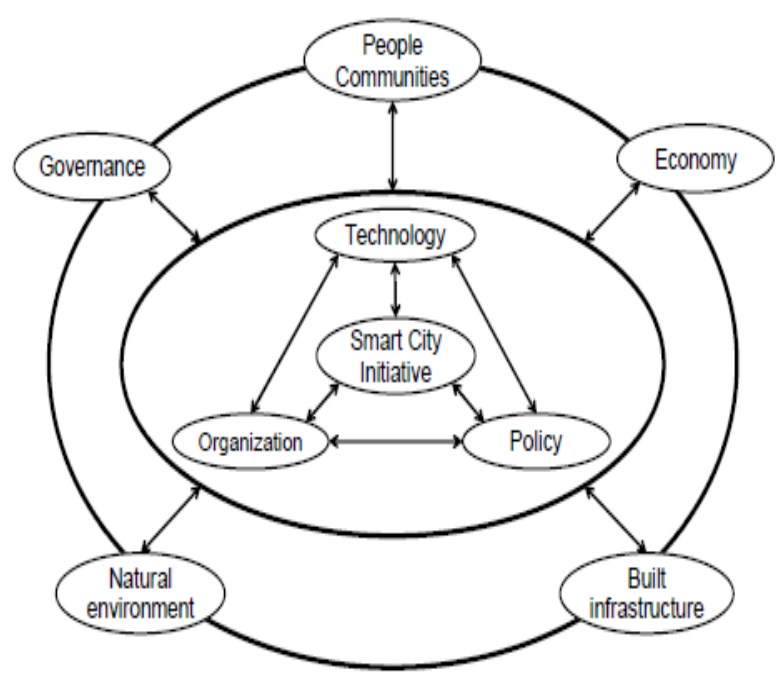

Fig.3.smartcity framework

\section{THE RESULT OF THEMATIC ANALYSIS}

The thematic analysis main intention to implement in this concept is repeated with worth able themes of the designed able to maintain certain level of smart cities and in here interlinked with internet of things, big data analytics and sensors are maintained balanced operations with nature of the environments. The theme of the target and also interface with advanced sensor technologies advanced computing systems, data collection and transfer platforms.

\section{INFRASTRUCTURE COMMUNICATION TECHNOLOGY}

\section{BIG DATA ANALYSIS ON DATA COLLECTION}

The collection of information or data sensing with sensors this is the present and future developing technology for smart city development process. Not only sensors and also consider the advanced computing systems and internet of things. The almost all information's are observed using monitor, based on this easy to understand and analyzed the actual positions and executed plan of the smart cities, these all information's are gathered digital sensors and also observations, transactions and the moments are stored in the process of organization, the collection of sensor data is transformed in various manners because of data processing, the data is mingle with data mining methods, visualization techniques. The satellite communication technology is one of the best ways to choose smart city developments, based on this using remote sensing techniques and capture and sensing the wanted information with internet of things. According to these calculations the big data analytics provide solutions for data processing. And also 
the data mining and machine learning techniques are gives good solutions on wide spectrum data sensing applications. Such type of cities monitoring, observations and information gathering all are done automatically with sensors. The advanced gaining and sensing technology is improved because of city developments that are infrared, laser devices, sound, light, heat, pressure, etc. Based on this nature environments operating devices and monitoring remote devices are available cameras, galvanometers, laser scanners, FID, GPS, radar guns and automation devices. Using this technology provides good opportunities to data monitoring functions and also operate effectively, this is provide a special way for smart city developments. The particular structural improvement is based on the smart electronics information systems like embedded systems and wireless communications. The intelligence information organisations are depends upon this smart electronics systems. Based on environmental and smart city infrastructure security, solutions and for information's its very useful.

\section{RADIO FREQUENCY ID TAGS WITH IOT AND AUTOMATED BIGDATA}

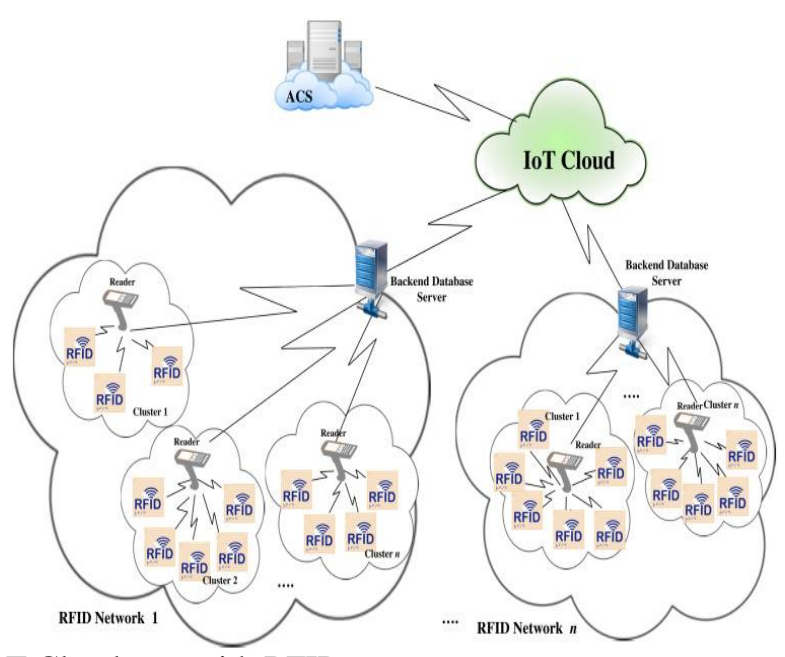

Fig.4. IoT Cloud tags with RFID

The tagging components are always attached with human's life. In here the tagging with iot devices and RFID components, the sensors are sensing or collecting the information from human's environment and sending the data to informational world. And its independently collecting the information using this technology it's not easy to modify the real time information. the computing devices all are minimized with nano technology, sensors and remaining devices all are very small size and easy to fix any place and easy to collect the information from any place or climate. The internet of things information analyze, systematically extract the information automatically because of automated big data analytics, this is the one of the advanced technology in data processing and storage systems. This automated big data analytics is merged with internet of things and computing systems, best option to choose smart city developments and planning's.

\section{DATA MANAGEMENT PLATFORMS.}

The information management systems are most important developing element for information and communication technology in smart city development. Best and advanced platforms are used for smart city information store and process purpose, that is hadoop is used for map reduce, spark and NoSQL -database system management. The big data analytics is widely used for large scale applications. In here considering various types of data processing platforms based on different sectors like data storage, data analysis, data management and bulk data sets depending upon based on different operations. Mainly concentrated plat from is here hadoop map reduce platform, this platform easily to maintain urban data and also have benefits are load balancing, reducing cost and processing power.

\section{CLOUD COMPUTING}

The cloud computing expressed in different applications and different applications. The internet communication technology researchers are working with various agencies and organisations, like education, hospitality and government. The cloud computing is data processing is done with internet controlled by user. The cloud computing is provided a three valuable real time services as one is software as a service is providers software and software development platform, second, platform as a service and third one is infrastructure as a service or customers is data storage management, virtual server, processors and network as resources. The big data analytics now days operated in cloud computing, because of these operations in here to introduce two services as platform as service and infrastructure as a service based on these services, the big data analytics combine to operate with cloud computing. The internet based computing systems are most advantage to smart city information to improve storage capacity, network bandwidth and energy.

\section{FOG / EDGE COMPUTING}

The fog or edge computing both are alternative of the cloud computing and also this computing model as well as merge with internet of things and big data analytics. The cloud computing and fog or edge computing both computing are operate as same operations like storage, applications and both are have backbone same as internet. in here the application is on smart city development and this is have large geographical structure, so its not easy to maintain cloud computing for this fog or edge computing is best for large geographical areas. The fog or edge computing is increasing the storage information because of improved bandwidth with internet of 
things. The source to computing systems information collection is same as both, but the quality is providing fog or edge computing. Now a days the fog network is responds with internet of things like mobile phones and real time using devices.

\section{SMART CITY DEVELOPEMENT APPLICATIONS : BIG DATA WITH IOT}

When the big data analytics is associated with internet of things it is easy to reach the goal of smart city developments. Why means, in this not one place to collect the information, collected different places, areas, organizations and controlling areas. This type of large data is collected with advanced internet of things and data stored, extracted and transfer in particular manner with big data analytics.

The internet of things are plays a main role to control the traffic in city roads using sensors, digital cameras and tracking systems. In no parking places also it's easy to control the traffic system. And also it is useful for toll gate areas to heavy traffic control purpose and large vehicle warning signals also provide to control. In smart city developments one of the main elements are pollution is also sensing and give the information to big data analytics.

The big data with internet of things used this technology easy to save the supply of energy. In smart city highway road lights, city public places LED lights these are control this technology without manpower within less time, using this technique save the maximum power. And also homemade needs also present days all associated with automated systems. The internet of things plays a main role to control the sound pollution and air pollution, using these sensors easy to find out pollution data, the factories are providing too much of pollution based on this internet of things easy to solve some more of air pollution and also sound pollution. Using this loop sensors information easy to find out the number cars flowing and emissions.

The smart city using the internet of things like system sensor and actuators to control and monitoring the mechanical machineries, electrical systems and electronic equipments are used in building constructions or maintained, industrial, public and smart buildings. The smart building are automatically to control with sensors, cameras, storage devices and easy to monitor the information. the mechanical and electrical systems are control the heat, ventilation and air conditions in large industries, it is also automatically control the all parameters and transferred to the information to database. The smart city development process constructs the bridges and tunnels and railway tracks, these all are monitoring and controlling used with internet of things. Used this technology to improve the security, reduce effective cost and service quality

\section{CHALLENGES: BIG DATA WITH IOT}

The internet of things and big data analytics are more demand in various applications and this is the comfortable technology to use and smart city development. Used this technology more challenges are occurred with smart city development process, that all are to overcome, design the structure, development, and data processing applications. These all issues are occurred scientific, computational and analytical way. The design constraints is start with problem and end with particular design limitations with design solution, in this process engineers are connected all issues with this, and understand with analysis. The data analytics is systematically introduce the statistical and logical techniques to get the evaluate data. The internet of things is concentrated particular area to sense or capture the exact information. The smart city has different services, structures and industries all this information integrates with fog or edge computing. The main challenges are occurred with big data analytics from environment information is too large, very different and change the information with fraction of seconds. In here very difficult to maintain urban information to manage, integrate process and analyze and evaluate to get exact knowledgeable data. And also have technical, organizational, institutional and structural issues are associated with implantation in big data analytics across the all domains.

The fig.5. Having controversies fig.5. the aim to guide and facilitate sense making understand for data storage and secure applications, these associated systematically with internet of things for advanced environmental city automation and security purpose. In this application using big data analytics is having limited access, some situations requires humans information or organizational information to choose between alternatives that must be evaluated as right. All the things are considered, understanding, misusing, and expanding the accessible calculation, examination, the board capabilities related with huge information investigations as per as organizations, devices, standards, ideal models, techniques, dangers, incredible open doors should be acknowledged as far as improving, outfitting, and incorporating urban frameworks and in this manner encouraging joint effort, coordination, and coupling among urban spaces through information driven and applications to progress natural maintainability with regards to keen supportable urban areas. The big data analytics is safe to use as long in urban analytics, this strategically implemented for data monitoring, situational analysis, observations, and planning for smart city development, the infrastructure communication technology is gradually improve the working functionality to long run applications. 
International Journal of Engineering Applied Sciences and Technology, 2020

Vol. 4, Issue 11, ISSN No. 2455-2143, Pages 113-122

Published Online March 2020 in IJEAST (http://www.ijeast.com)

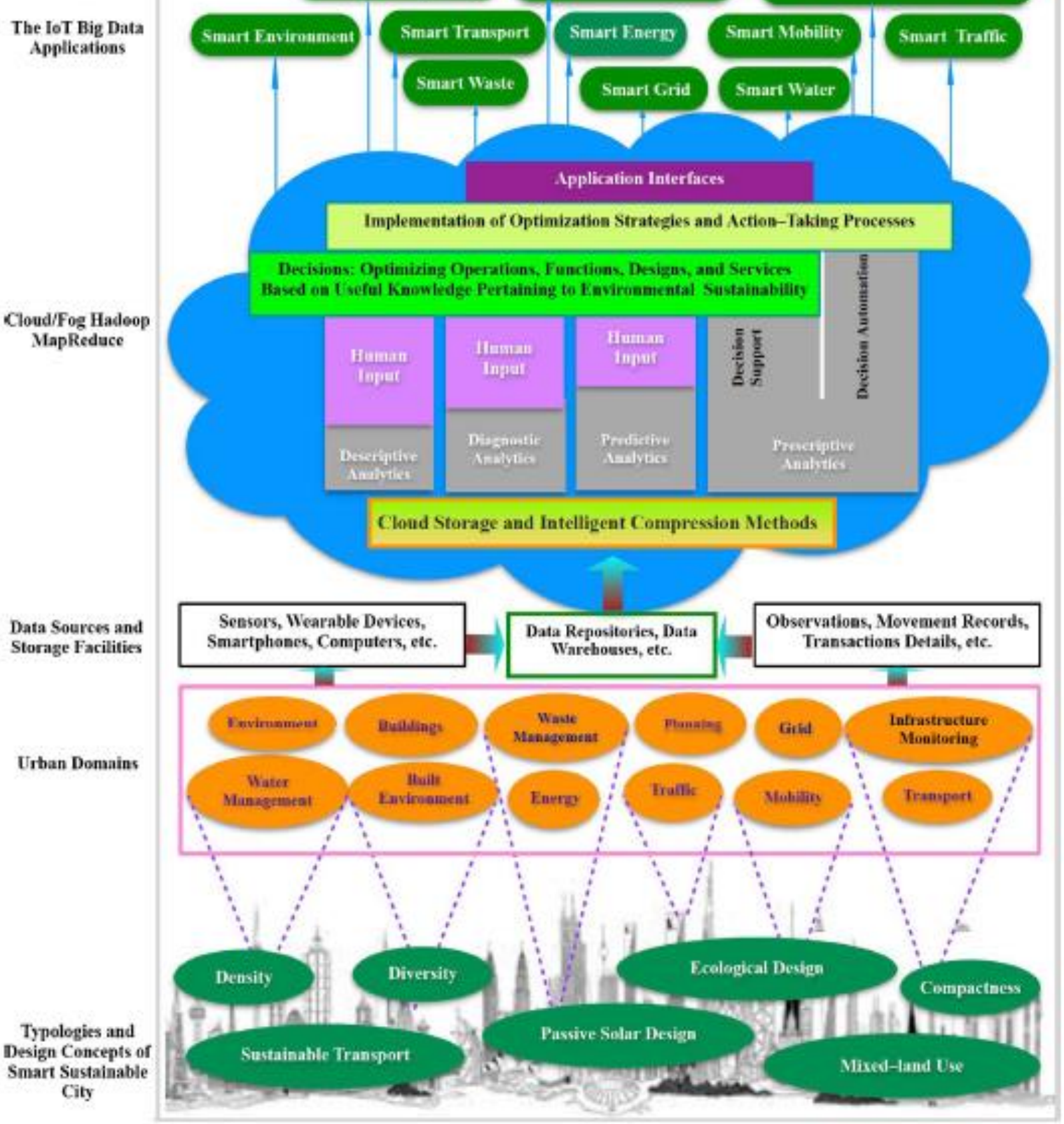

Fig.5. Big Data with IoT Infrastructure Communication Technology analytical structure. 


\section{CONCLUSION}

The combination of big data analytics and internet if things are given best results for smart city infrastructure, environmental issues and communicating services improved. The fog or edge computing giving $10 \%$ to $15 \%$ best results on large geo structural data storage, quality of data and management than cloud computing. The internet of things and big data analytics are integrated for future developments both ecologically and technologically for many cities. The integrated technology big data with IoTs are provide advanced security for smart city planning management and quality life for citizens'. The advanced sensing technology is automatically sense the information and monitor, analyze and reduce the issues easily, this system totally based on infrastructure communication technology. The large geo graphical city infrastructure Hadoop, Map Reduce platforms are good choice to choose than other platforms.

\section{REFERENCE}

[1] T. Savolainen, J. Soininen and B. Silverajan, "IPv6 Addressing Strategies for IoT," IEEE Sensors Journal, vol. 13, no. 10, pp. 3511-3519, Oct. 2013.

[2] J. W. Hui and D. E. Culler, "IPv6 in Low-Power Wireless Networks," Proceedings of the IEEE, vol. 98, no. 11, pp. 1865-1878, Nov. 2010.

[3] J. Olsson, "6LoWPAN demystified," Texas Instruments, 2014.

[4] F. T. Mamo and A. Sikora, "Implementation of standardized 6LoWPAN based application layer protocols," 2015 IEEE 8th International Conference on Intelligent Data Acquisition and Advanced Computing Systems: Technology and Applications (IDAACS), Warsaw, 2015, pp. 817-822.

[5] C. Gomez, J. Paradells, C. Bormann and J. Crowcroft, "From 6LoWPAN to 6Lo: Expanding the Universe of IPv6Supported Technologies for the Internet of Things," IEEE Communications Magazine, vol. 55, no. 12, pp. 148-155, Dec. 2017.

[6] Internet Protocol for Smart Objects (IPSO) Alliance, RPL: The IP routing protocol designed for low power and lossy networks, 2011.

[7] N. Accettura, L. A. Grieco, G. Boggia and P. Camarda, "Performance analysis of the RPL Routing Protocol," 2011 IEEE International Conference on Mechatronics, Istanbul, 2011, pp. 767-772.

[8] A. Aijaz, H. Su and A. H. Aghvami, "CORPL: A Routing Protocol for Cognitive Radio Enabled AMI Networks," IEEE Transactions on Smart Grid, vol. 6, no. 1, pp. 477-485, Jan. 2015.

[9] M. Lauridsen, H. Nguyen, B. Vejlgaard, I. Z. Kovacs, P. Mogensen and M. Sorensen, "Coverage Comparison of GPRS, NB-IoT, LoRa, and SigFox in a 7800 km2 Area," 2017 IEEE 85th Vehicular Technology Conference (VTC Spring), Sydney, NSW, 2017, pp. 1-5.
[10] M. Elsaadany, A. Ali and W. Hamouda, "Cellular LTE-A Technologies for the Future Internet-of-Things: Physical Layer Features and Challenges," IEEE Communications Surveys \& Tutorials, vol. 19, no. 4, pp. 2544-2572, Fourthquarter 2017.

[11] S. Basagni, C. Petrioli, R. Petroccia and D. Spaccini, "Channel-aware routing for underwater wireless networks," 2012 Oceans - Yeosu, Yeosu, 2012, pp. 1-9.

[12] Z. Zhou, B. Yao, R. Xing, L. Shu and S. Bu, "E-CARP: An Energy Efficient Routing Protocol for UWSNs in the Internet of Underwater Things," IEEE Sensors Journal, vol. 16, no. 11, pp. 4072-4082, June, 2016.

[13] P. K. Wali and D. Das, "A novel access scheme for IoT communications in LTE-Advanced network," 2014 IEEE International Conference on Advanced Networks and Telecommuncations Systems (ANTS), New Delhi, 2014, pp. 16.

[14] IEEE Standard for a Convergent Digital Home Network for Heterogeneous Technologies Amendment 1: Support of New MAC/PHYs and Enhancements, IEEE Std 1905.1a-2014 (Amendment to IEEE Std 1905.1- 2013), vol., no., pp.1-52, Feb. 202015

[15] D. Wobschall, "IEEE 1451-a universal transducer protocol standard," 2007 IEEE Autotestcon, Baltimore, MD, 2007, pp. 359-363.

[16] C. Dai and Z. Wang, "A Flexible Extension of WSDL to Describe NonFunctional Attributes," 2010 2nd International Conference on E-business and Information System Security, Wuhan, 2010, pp. 1-4.

[17] G. Moritz, F. Golatowski and D. Timmermann, "A Lightweight SOAP over CoAP Transport Binding for Resource Constraint Networks," 2011 IEEE Eighth International Conference on Mobile Ad-Hoc and Sensor Systems, Valencia, 2011, pp. 861-866.

[18] G. L. D. Santos, V. T. Guimaraes, G. D. C. Rodrigues, L. Z. Granville and L. M. R. Tarouco, "A DTLS-based security architecture for the Internet of Things," 2015 IEEE Symposium on Computers and Communication (ISCC), Larnaca, 2015, pp. 809-815.

[19] R. Behrens and A. Ahmed, "Internet of Things: An endto-end security layer," 2017 20th Conference on Innovations in Clouds, Internet and Networks (ICIN), Paris, 2017, pp. 146149.

[20] G. Peralta, M. Iglesias-Urkia, M. Barcelo, R. Gomez, A. Moran and J. Bilbao, "Fog computing based efficient IoT scheme for the Industry 4.0," 2017 IEEE International Workshop of Electronics, Control, Measurement, Signals and their Application to Mechatronics (ECMSM), Donostia-San Sebastian, 2017, pp. 1-6.

[21] M. B. Yassein, M. Q. Shatnawi and D. Al-zoubi, "Application layer protocols for the Internet of Things: A survey," 2016 International Conference on Engineering \& MIS (ICEMIS), Agadir, 2016, pp. 1-4.

[22] M. Kirsche and R. Klauck, "Unify to bridge gaps: Bringing XMPP into the Internet of Things," 2012 IEEE 
International Conference on Pervasive Computing and Communications Workshops, Lugano, 2012, pp. 455-458.

[23] C. Bormann, A. P. Castellani and Z. Shelby, "CoAP: An Application Protocol for Billions of Tiny Internet Nodes," IEEE Internet Computing, vol. 16, no. 2, pp. 62-67, MarchApril 2012.

[24] J. B. Postel, "User datagram protocol; RFC-768," Internet Requests for Comments (768), Aug. 1980. 32

[25] S. Vinoski, "Advanced Message Queuing Protocol," IEEE Internet Computing, vol. 10, no. 6, pp. 87-89, Nov.-Dec. 2006.

[26] M. Chernyshev, Z. Baig, O. Bello and S. Zeadally, "Internet of Things (IoT): Research, Simulators, and Testbeds," IEEE Internet of Things Journal, vol. PP, no. 99, pp. 1-1.

[27] G. D'Angelo, S. Ferretti and V. Ghini, "Simulation of the Internet of Things," 2016 International Conference on High Performance Computing \& Simulation (HPCS), Innsbruck, 2016, pp. 1-8.

[28] T. Grass C. Allande, A. Armejach, A. Rico, E. Ayguade, J. Labarta, M. Valero, M. Casas, and M. Moreto, "MUSA: A Multi-level Simulation Approach for Next-Generation HPC Machines," SC16: International Conference for High Performance Computing, Networking, Storage and Analysis, Salt Lake City, UT, 2016, pp. 526-537.

[29] Y. Zhao, H. Yu and G. Liang, "NS3-based simulation system in heterogeneous wireless network," 11th International Conference on Wireless Communications, Networking and Mobile Computing (WiCOM 2015), Shanghai, 2015, pp. 1-6.

[30] F. Osterlind, A. Dunkels, J. Eriksson, N. Finne and T. Voigt, "CrossLevel Sensor Network Simulation with COOJA," 31st IEEE Conference on Local Computer Networks, Tampa, FL, 2006, pp. 641-648.

[31] I. Muscalagiu, C. Illes and H. E. Popa, "Large scale multi-agent-based simulation using NetLogo for the multirobot exploration problem," 2013 11th IEEE International Conference on Industrial Informatics (INDIN), Bochum, 2013, pp. 325-330.

[32] X. Zeng, S. K. Garg, P. Strazdins, P. Jayaraman, D. Georgakopoulos and R. Ranjan, "IOTSim: a Simulator for Analysing IoT Applications," Journal of System Architecture, Elsevier, vol. 72, issue C, pp. 93-107, Jan. 2017.

[33] A.F. Haryadi, J. Hulstijn, A. Wahyudi, H. van der Voort, M. Janssen, Antecedents of big data quality: An empirical examination in financial service organizations, in: Big Data (Big Data), 2016 IEEE International Conference on, IEEE, 2016, pp. 116-121.

[34] J. LeFevre, R. Liu, C. Inigo, L. Paz, E. Ma, M. Castellanos, M. Hsu, Building the enterprise fabric for big data with vertica and spark integration, in: Proceedings of the 2016 International Conference on Management of Data, ACM, 2016, pp. 63-75.

[35] Y. Wang, L. Kung, W.Y.C. Wang, C.G. Cegielski, Integrated big data analytics-enabled transformation model: Application to health care, Inf. Manage. (2017).
[36] G. Press, Cleaning Big Data: Most Time-Consuming, Least Enjoyable Data Science Task, Survey Says, Online, 2016.

[37] P. Wiener, M. Stein, D. Seebacher, J. Bruns, M. Frank, V. Simko, S. Zander, J. Nimis, Biggis: a continuous refinement approach to master heterogeneity and uncertainty in spatio-temporal big data (vision paper), in: Proceedings of the 24th ACM SIGSPATIAL International Conference on Advances in Geographic Information Systems, ACM, 2016, p. 8.

[38] B. Ong, R. Wen, A.N. Zhang, Data blending in manufacturing and supply chains, in: Big Data (Big Data), 2016 IEEE International Conference on, IEEE, 2016, pp. 3773-3778.

[39] D. García-Gil, J. Luengo, S. García, F. Herrera, Enabling Smart Data: Noise filtering in Big Data classification, arXiv preprint arXiv:1704.017702017.

[40] M. Bai, X. Wang, J. Xin, G. Wang, An efficient algorithm for distributed density-based outlier detection on big data, Neurocomputing 181 (2016) 19-28.

[41] L. Stojanovic, M. Dinic, N. Stojanovic, A. Stojadinovic, Big-data-driven anomaly detection in industry (4.0): An approach and a case study, in: Big Data (Big Data), 2016 IEEE International Conference on, IEEE, 2016, pp. 16471652.

[42] M. Niño, F. Sáenz, J.M. Blanco, A. Illarramendi, Requirements for a big data capturing and integration architecture in a distributed manufacturing scenario, in: Industrial Informatics (INDIN), 2016 IEEE 14th International Conference on, IEEE, 2016, pp. 1326-1329.

[43] R. Wang, D. Sun, G. Li, M. Atif, S. Nepal, Logprov: Logging events as provenance of big data analytics pipelines with trustworthiness, in: IEEE Conference on Big Data, 2016.

[44] K. Yamanishi, K. Miyaguchi, Detecting gradual changes from data stream using MDL-change statistics, in: Big Data (Big Data), 2016 IEEE International Conference on, IEEE, 2016, pp. 156-163.

[45] X. Cheng, L. Fang, X. Hong, L. Yang, Exploiting mobile big data: Sources, features, and applications, IEEE Network 31 (1) (2017) 72-79.

[46] A.A.F. Saldivar, C. Goh, W.-n. Chen, Y. Li, Selforganizing tool for smart design with predictive customer needs and wants to realize industry 4.0, in: Evolutionary Computation (CEC), 2016 IEEE Congress on, IEEE, 2016, pp. 5317-5324. 